\title{
Fault Detection Filter Design for Stochastic Systems with Mixed Time-Delays and Parameter Uncertainties
}

\author{
Liyuan Hou, ${ }^{1}$ Shouming Zhong, ${ }^{2,3}$ Hong Zhu, ${ }^{1}$ Yong Zeng, ${ }^{1}$ and Lin Shi ${ }^{1}$ \\ ${ }^{1}$ School of Automation Engineering, University of Electronic Science and Technology of China, Chengdu 611731, China \\ ${ }^{2}$ School of Mathematical Sciences, University of Electronic Science and Technology of China, Chengdu 611731, China \\ ${ }^{3}$ Key Laboratory for Neuroinformation of Ministry of Education, University of Electronic Science and Technology of China, \\ Chengdu 611731, China
}

Correspondence should be addressed to Liyuan Hou; liyuanhou@163.com

Received 29 May 2013; Accepted 5 August 2013

Academic Editors: A. Bellouquid, A. J. Kearsley, and C.-H. Lien

Copyright (c) 2013 Liyuan Hou et al. This is an open access article distributed under the Creative Commons Attribution License, which permits unrestricted use, distribution, and reproduction in any medium, provided the original work is properly cited.

This paper purposes the design of a fault detection filter for stochastic systems with mixed time-delays and parameter uncertainties. The main idea is to construct some new Lyapunov functional for the fault detection dynamics. A new robustly asymptotically stable criterion for the systems is derived through linear matrix inequality (LMI) by introducing a comprehensive different LyapunovKrasovskii functional. Then, the fault detection filter is designed in terms of linear matrix inequalities (LMIs) which can be easily checked in practice. At the same time, the error between the residual signal and the fault signal is made as small as possible. Finally, an example is given to illustrate the effectiveness and advantages of the proposed results.

\section{Introduction}

Stochastic systems have strong practical relevance in mechanical systems, economics, systems with human operators, and other engineering areas. Meanwhile, the filtering problem has many applications in the areas of signal processing, signal estimation, pattern recognition, and many practical control systems. Therefore, the filter design problems and the stochastic systems have become important areas of research and received great attention over the past few years [1-6].

With the rising demand for higher safety and increasing demand for higher performance in the modern industries, the research on fault detection for dynamic systems has received more and more attention during the past two decades, like model-based schemes [7], knowledge-based approaches [8], and signal-based methods [9], and so forth. Particularly, the problem of model-based fault detection has been an active research area among them. The basic objective of model-based fault detection is to construct the residual generator, and to determine the residual evaluation function and the threshold. An alarm of fault will be generated, when the value of the evaluation function is greater than the stated threshold. However, randomly occurring nonlinearities and the existence of unknown inputs may seriously affect the performance of model-based fault detection systems. Therefore, robustness issue plays an important role in the application of model-based fault detection schemes. Increasing the robustness of residual to unknown inputs and modelling errors and enhancing the sensitivity to faults are of prime importance in designing a model-based fault detection system. Among different methods for fault detection, the model-based approach has been widely used in recent years. So far, the problem of fault detection has been thoroughly investigated for a variety of systems including uncertain systems [2, 10-14], time-delay systems [15-17], and Markovian jump linear systems [18].

Besides, time-delay is one of the major sources of instability and poor performance of a practical control system. And many results on stochastic systems with time delays have been reported in the literature [10-12] and the references therein. There is a need to discuss the distributed delays that occur very often in practical systems. The engineering significance of distributed delays has been widely recognized, and a number of corresponding results have been published; see, for example, [15]. The distributed delays in the discrete-time setting, on the other hand, have received little attention. It is well known that nonlinearities exist universally in practical 
systems, and therefore nonlinear control has been an ever hot topic in the past few decades.

As far as we know, the delay-dependent criteria on fault detection filter design for delayed stochastic systems with parameter uncertainties have not been fully studied, which is still open. Motivated by the above discussion and in order to obtain less conservative results, we choose an appropriate new Lyapunov functional and establish a new integral inequality in the stochastic setting. What is more, because we have carefully considered the ranges for the timevarying delays, our criteria can be applicable to both fast and slow time-varying delays. The stability criteria obtained are in terms of linear matrix inequalities (LMIs) which can be checked efficiently via the LMI toolbox. Finally, a numerical example is also given to demonstrate the effectiveness and advantages of our theoretical results.

Notations. The notations used throughout the paper are fairly standard. The superscript " $T$ " stands for matrix transposition; $R^{n}$ denotes the $n$-dimensional Euclidean space; $R^{n \times m}$ is the set of all $n \times m$ real matrices; the notation $P>0$ means that $P$ is a positive definite matrix; $I$ and 0 represent identity matrix and zero matrix, respectively; and $\operatorname{diag}(\cdot)$ denotes the diagonal matrix. The vector norm is taken to be Euclidean, and the matrix norm is the corresponding induced one. $E\{\cdot\}$ denotes the expectation operator. $\mathscr{L}_{2}[0,+\infty)$ is the space of square-integrable vector function over $[0,+\infty)$. In symmetric block matrices, we use an asterisk $(*)$ to represent a term that is induced by symmetry. Matrices, if their dimensions are not explicitly stated, are assumed to be compatible for algebraic operations.

\section{Problem Formulation and Some Preliminaries}

The simplest and most fundamental case considered is the problem of quadratic stabilization for the following system:

$$
\begin{gathered}
x(k+1)=A x(k)+B u(k) \\
y(k)=C x(k),
\end{gathered}
$$

where $x(k) \in R^{n}$ is the state and $u(k)$ is the control input. Then, we add discrete time-delays $\tau_{i}(k),(i=1,2, \ldots, q)$, while $d(d=1,2, \ldots, \infty)$ describe the distributed time-delays. In addition, we are motivated to study the fault detection problem for a class of discrete-time systems involving stochastic time-delay, such that we consider the following stochastic time-delay system:

$$
\begin{aligned}
x(k+1)= & A x(k)+A_{1} \sum_{i=1}^{q} \alpha_{i}(k) x\left(k-\tau_{i}(k)\right) \\
& +\beta(k) A_{2} \sum_{d=1}^{\infty} \mu_{d} x(k-d)+\gamma(k) g(k, x(k)) \\
& +D_{1} w(k)+G f(k)
\end{aligned}
$$

$$
\begin{gathered}
y(k)=C x(k)+D_{2} w(k)+H f(k) \\
x(k)=\phi(k), \quad \forall k \in \mathbb{Z}^{-}
\end{gathered}
$$

where $x(k) \in R^{n}$ is the state; $y(k) \in R^{m}$ is the process output; $w(k) \in R^{p}$ is the unknown input which belongs to $\mathscr{L}_{2}[0, \infty)$; $f(k) \in R^{l}$ is the fault to be detected; $A, A_{1}, A_{2}, C, D_{1}$, $D_{2}$, and $G$ are real known constant matrices with compatible dimensions. The discrete time-delay $\tau_{i}(k)(i=1,2, \ldots, n)$ is a time-varying differentiable function satisfying $\tau_{m} \leq$ $\tau_{i}(k) \leq \tau_{M}$, while $d(d=1,2, \ldots, \infty)$ denotes the discrete time-delays, and the constant $\mu_{d} \geq 0$ satisfies the following convergence conditions $\bar{\mu}:=\sum_{d=1}^{\infty} \mu_{d} \leq \sum_{d=1}^{\infty} d \mu_{d}<\infty$, $\widehat{\mu}:=\sum_{d=1}^{\infty} \mu_{d}^{2}<\infty$.

Assumption 1. The nonlinear function $g(k, x(k)) \in R^{n}$ satisfies the following condition: for any $x, y \in R,(x \neq y)$,

$$
\widehat{g}_{i}^{-} \leq \frac{g_{i}(x)-g_{i}(y)}{x-y} \leq \widehat{g}_{i}^{+},
$$

where $\widehat{g}_{i}^{-}, \hat{g}_{i}^{+}(i=1,2, \ldots, n)$ are constants.

Assumption 2. The stochastic variables $\alpha_{i}(k)\left(i=1,2, \ldots n_{1}\right)$, $\beta(k)$, and $\gamma(k)$ and mutually uncorrelated Bernoulli distributed white sequences that account for, respectively, the phenomena of randomly occurring discrete time-delays, distribute time-delays, and nonlinearities. A natural assumption on the sequences $\alpha_{i}(k)\left(i=1,2, \ldots n_{1}\right), \beta(k)$, and $\gamma(k)$ is made as follows:

$$
\begin{gathered}
\operatorname{Prob}\left\{\alpha_{i}(k)=1\right\}=\mathbb{E}\left\{\alpha_{i}(k)\right\}=\bar{\alpha}_{i}, \\
\operatorname{Prob}\left\{\alpha_{i}(k)=0\right\}=1-\bar{\alpha}_{i}, \\
\operatorname{Prob}\{\beta(k)=1\}=\mathbb{E}\{\beta(k)\}=\bar{\beta}, \\
\operatorname{Prob}\{\beta(k)=0\}=1-\bar{\beta}, \\
\operatorname{Prob}\{\gamma(k)=1\}=\mathbb{E}\{\gamma(k)\}=\bar{\gamma}, \\
\operatorname{Prob}\{\gamma(k)=0\}=1-\bar{\gamma} .
\end{gathered}
$$

In a networked environment, it is quite common that the measurements $y(k)$ of the system are quantized during the signal transmission. Let us denote the quantizer as $h(\cdot)=$ $\left[h_{1}(\cdot), h_{2}(\cdot), \ldots, h_{m}(\cdot)\right]^{T}$ which is symmetric, that is, $h_{j}(-v)=$ $-h_{j}(\nu), j=1, \ldots, m$.

The map of the quantization process is $\widehat{y}(k)=h(y(k))=$ $\left[h_{1}\left(y^{(1)}(k)\right), h_{2}\left(y^{(2)}(k)\right), \ldots, h_{m}\left(y^{(m)}(k)\right)\right]^{T}$. In this paper, we are interested in the logarithmic static and time-invariant quantizer. For each, the set of quantization levels is described by $\mathcal{U}_{j}=\left\{ \pm \widehat{\mu}_{i}^{(j)}, \widehat{\mu}_{i}^{(j)}=\chi_{j}^{j} \widehat{\mu}_{0}^{(j)}, i=0, \pm 1, \pm 2, \ldots\right\} \cup\{0\}, 0<\chi_{j}<$ $1, \widehat{\mu}_{0}^{(j)}>0$, and each of the quantization levels corresponds to a segment, such that the quantizer maps the whole segment 
to this quantization level. According to [19], the quantizer is given by

$$
\begin{aligned}
& h_{j}\left(y^{(j)}(k)\right) \\
& \quad= \begin{cases}\widehat{\mu}_{i}^{(j)}, & \frac{1}{1+\delta_{j}} \widehat{\mu}_{i}^{(j)} \leqslant y^{(j)}(k) \leqslant \frac{1}{1-\delta_{j}} \widehat{\mu}_{i}^{(j)} \\
0, & y^{(j)}(k)=0 \\
-h_{j}\left(-y^{(j)}(k)\right), & y^{(j)}(k)<0,\end{cases}
\end{aligned}
$$

where $\delta_{j}=\left(1-\chi_{j}\right)\left(1+\chi_{j}\right)$, such that $h_{j}\left(y^{(j)}(k)\right)=$ $\left(I+\Delta_{k}\right) y^{(j)}(k)$ with $\left|\Delta_{k}^{(j)}\right| \leqslant \delta_{j}$; the measurements with quantization effect can be expressed as

$$
\begin{aligned}
\widehat{y}(k)= & \left(I+\Delta_{k}\right) y(k) \\
= & \left(I+\Delta_{k}\right) C x(k)+\left(I+\Delta_{k}\right) D_{2} \omega(k) \\
& +\left(I+\Delta_{k}\right) H f(k),
\end{aligned}
$$

with $\Delta_{k}=\operatorname{diag}\left(\Delta_{k}^{(1)}, \ldots, \Delta_{k}^{(m)}\right)$.

Consider the following full-order fault detection filter for system (2):

$$
\begin{gathered}
\widehat{x}(k+1)=A_{f} \widehat{x}(k)+B_{f} \widehat{y}(k), \\
r(k)=C_{f} \widehat{x}(k)+D_{f} \hat{y}(k),
\end{gathered}
$$

where $\widehat{x}(k) \in R^{n}$ is the filter state vector, $r(k) \in \mathbb{R}^{l}$ is the so called residual that is compatible with the fault vector $f(k)$, and $A_{f}, B_{f}$, and $C_{f}$ are appropriately dimensioned filter matrices to be designed.

From (2), (5), and (7), we can obtain the following filtering error system:

$$
\begin{aligned}
\widetilde{x}(k+1)= & (\widetilde{A}+\Delta A) \widetilde{x}(k) \\
& +\sum_{i=1}^{q}\left(\widetilde{A}_{1 i}+\widetilde{A}_{1 i}(k)\right) \widetilde{x}\left(k-\tau_{i}(k)\right) \\
& +\left(\widetilde{A}_{2}+\widetilde{A}_{2}(k)\right) \sum_{d=1}^{\infty} \mu_{d} \tilde{x}(k-d) \\
& +(\bar{\gamma}+\widetilde{\gamma}(k)) \widetilde{I} g(k, x(k))+(\widetilde{D}+\Delta D) v(k) \\
\widetilde{r}(k)= & (\widetilde{C}+\Delta C) \tilde{x}(k)+\left(\widetilde{D}_{f}+\Delta D_{f}\right) v(k) \\
& \tilde{x}(k)=\widetilde{\phi}(k), \quad \forall k \in\left[-\tau_{M}, 0\right],
\end{aligned}
$$

with

$$
\begin{aligned}
& \tilde{x}(k)=\left[x^{T}(k), \widehat{x}^{T}(k)\right]^{T}, \\
& \tilde{\phi}^{T}(k)=\left[\phi^{T}(k), 0^{T}\right], \\
& \widetilde{r}(k)=r(k)-f(k), \quad v(k)=\left[\omega^{T}(k), f^{T}(k)\right]^{T}, \\
& \widetilde{A}=\left(\begin{array}{cc}
A & 0 \\
B_{f} C & A_{f}
\end{array}\right), \quad \Delta A=\left(\begin{array}{cc}
0 & 0 \\
B_{f} \Delta_{k} C & 0
\end{array}\right), \\
& \widetilde{A}_{1 i}=\left(\begin{array}{cc}
\bar{\alpha}_{i} A_{1} & 0 \\
0 & 0
\end{array}\right), \quad \widetilde{A}_{1 i}(k)=\left(\begin{array}{cc}
\widetilde{\alpha}_{i}(k) A_{1} & 0 \\
0 & 0
\end{array}\right), \\
& \widetilde{A}_{2}=\left(\begin{array}{cc}
\bar{\beta} A_{2} & 0 \\
0 & 0
\end{array}\right), \quad \widetilde{A}_{2}(k)=\left(\begin{array}{cc}
\widetilde{\beta}(k) A_{2} & 0 \\
0 & 0
\end{array}\right) \text {, } \\
& \widetilde{I}=\left(\begin{array}{l}
I \\
0
\end{array}\right), \quad \widetilde{D}=\left(\begin{array}{cc}
D_{1} & G \\
B_{f} D_{2} & B_{f} H
\end{array}\right), \\
& \Delta D=\left(\begin{array}{cc}
0 & 0 \\
B_{f} \Delta_{k} D_{2} & B_{f} \Delta_{k} H
\end{array}\right), \quad \widetilde{C}=\left(\begin{array}{lll}
D_{f} C & C_{f}
\end{array}\right), \\
& \Delta C=\left(\begin{array}{lll}
D_{f} \Delta_{k} C & 0
\end{array}\right), \quad \widetilde{D}_{f}=\left(\begin{array}{ll}
D_{f} D_{2} & D_{f} H-I
\end{array}\right), \\
& \Delta D_{f}=\left(\begin{array}{lll}
D_{f} \Delta_{k} D_{2} & D_{f} \Delta_{k} H
\end{array}\right), \quad \widetilde{\alpha}_{i}(k)=\alpha_{i}(k)-\bar{\alpha}_{i}, \\
& \tilde{\beta}(k)=\beta(k)-\bar{\beta}, \quad \tilde{\gamma}(k)=\gamma(k)-\bar{\gamma} .
\end{aligned}
$$

In order to conduct the stability analysis for the above systems, it is necessary to make the following definitions and lemmas.

Definition 3. Consider the system (8) with $v(k)=0$; it is said to be mean-square exponentially stable if for any initial conditions, there exist a $\delta>0$ and $0<\alpha<1$, such that $E\left\{|\widetilde{x}(k)|^{2}\right\} \leq \delta \alpha^{k} \sup _{i \in Z^{-}} E\left\{|\widetilde{\phi}(i)|^{2}\right\}, k \geq 0$. Moreover, if $\lim _{t \rightarrow \infty} E\left\{|x(k)|^{2}\right\}=0$, then the system is said to be robust asymptotically mean-square stable.

Definition 4. Given a scalar $\gamma>0$, the error system (8) is said to be mean-square robustly exponentially stable with the attenuation level $\gamma$ if the error system (8) with $v(k)=0$ is mean-square robustly exponentially stable, and under zero initial condition, the following is true for all nonzero $v(k)$ :

$$
\begin{gathered}
\sum_{k=0}^{+\infty} E\left\{\widetilde{r}^{T}(k) \widetilde{r}(k)\right\} \leq \gamma^{2} \sum_{k=0}^{\infty} E\left\{v^{T}(k) v(k)\right\} \\
\text { (i.e., } \left.\|e\|_{E_{\infty}} \leq \gamma\|v\|_{2}, \forall w(k) \in \mathscr{L}_{2}[0,+\infty)\right) .
\end{gathered}
$$

Correspondingly, system (7) is said to be stochastic $\mathscr{L}_{2}-\mathscr{L}_{\infty}$ filter of system (2).

We further adopt a residual evaluation stage including an evaluation function $J(k)$ and a threshold $J_{\text {th }}$ of the following form: $J(k)=\sum_{h=0}^{k} r(h) r(h)^{1 / 2}$ where $L$ denotes the maximum time step of the evaluation function. Based on (16), the occurrence of faults can be detected by comparing $J(k)$ with 
$J_{\text {th }}$ according to the following rule: $J(k)>J_{\text {th }}$ (with faults) alarm; $J(k)<=J_{\text {th }}$ no faults.

Remark 5. The parametric uncertainties $\Delta A, \Delta D, \Delta C$, and $\Delta D_{f}$ satisfy

$$
\left[\begin{array}{cc}
\Delta A & \Delta D \\
\Delta C & \Delta D_{f}
\end{array}\right]=\left[\begin{array}{c}
0 \\
B_{f} \\
D_{f}
\end{array}\right] F(k)\left[\Lambda C, 0, \Lambda D_{2}, \Lambda H\right],
$$

where $\Lambda=\operatorname{diag}\left\{\delta_{1}, \delta_{2}, \ldots, \delta_{m}\right\}$ and $F(k)=\Delta_{k} \Lambda^{-}$, and $F(k)$ satisfies $F^{T}(k) F(k) \leq I$.

Lemma 6. Let $M, N$, and $F$ be real matrices of appropriate dimensions with $F^{T} F \leq I$. Then, for any scalar $\epsilon>0$, one has

$$
M F N+(M F N)^{T} \leq \epsilon^{-1} M M^{T}+\epsilon N N^{T} .
$$

Lemma 7 (Schur complement). Given constant matrices $S_{1}$, $S_{2}$, and $S_{3}$, where $S_{1}=S_{1}^{T}$ and $S_{2}=S_{2}^{T}$, then $S_{1}+S_{3}^{T} S_{2}^{-1} S_{3}<0$ if and only if

$$
\left[\begin{array}{cc}
S_{1} & S_{3}^{T} \\
S_{3} & -S_{2}
\end{array}\right]<0, \quad \text { or } \quad\left[\begin{array}{cc}
-S_{2} & S_{3} \\
S_{3}^{T} & S_{1}
\end{array}\right]<0
$$

Lemma 8 (Liu et al. [5]). For any constant matrix $M \in R^{n \times n}$, $M=M^{T}>0, x_{i} \in R^{n}$, and constants $a_{i}>0(i=1,2, \ldots)$. If the series concerned is convergent, then one has

$$
\begin{aligned}
& -\left(\sum_{i=1}^{\infty} a_{i}\right) \sum_{i=1}^{\infty} a_{i} x_{i}^{T} M a_{i} x_{i}^{T} \\
& \leq-\left(\sum_{i=1}^{\infty} a_{i} x_{i}\right)^{T} M\left(\sum_{i=1}^{\infty} a_{i} x_{i}\right) .
\end{aligned}
$$

\section{Main Results}

In this section, we will investigate a sufficient condition on the performance analysis for the filter error system (8). And then, the solution to the fault detection filter design for the system (2) is listed as follows.

Theorem 9. For nominal system of (8) with given filter parameters and the index $\gamma>0$, the fault detection dynamics is robustly exponentially stable in mean square, if there exist constant $l>0$, positive matrices $P, Q, Q_{i}(i=1,2, \ldots, q)$, and any matrices $U$ with appropriate dimensions, such that the following LMIs hold:

$$
\begin{aligned}
& \Gamma=\left[\begin{array}{ccccccccc}
\Gamma_{11} & 0 & \cdots & 0 & 0 & \Gamma_{14} & 0 & \widetilde{C}^{T} & \widetilde{A}^{T} P \\
* & \bar{\alpha}_{1}\left(1-\bar{\alpha}_{1}\right) \widehat{A}_{1}^{T} P \widehat{A}_{1}-Q_{1} & \cdots & 0 & 0 & 0 & 0 & 0 & \widetilde{A}_{11}^{T} P \\
* & * & \ddots & \vdots & \vdots & \vdots & \vdots & \vdots & \vdots \\
* & * & * & \bar{\alpha}_{q}\left(1-\bar{\alpha}_{q}\right) \widehat{A}_{1}^{T} P \widehat{A}_{1}-Q_{q} & 0 & 0 & 0 & 0 & \widetilde{A}_{1 q}^{T} P \\
* & * & * & * & -\frac{Q}{\bar{\mu}} & 0 & 0 & 0 & \widetilde{A}_{2}^{T} P \\
* & * & * & * & * & \Gamma_{44} & 0 & 0 & \widetilde{\gamma}^{T} P \\
* & * & * & * & * & * & \Gamma_{55} & \widetilde{D}_{f}^{T} & \widetilde{D}^{T} P \\
* & * & * & * & * & * & * & -I & 0 \\
* & * & * & * & * & * & * & * & -P
\end{array}\right]<\text {, } \\
& \Gamma_{11}=-P+\sum_{i=1}^{q}\left(\tau_{M}-\tau_{m}+1\right) Q_{i}+\bar{\mu} Q+\widehat{\mu} \bar{\beta}(1-\bar{\beta}) \widehat{A}_{2}^{T} P \widehat{A}_{2}-\left[\begin{array}{cc}
U G_{1} & 0 \\
0 & 0
\end{array}\right], \\
& \Gamma_{14}=\left[\begin{array}{c}
U G_{2} \\
0
\end{array}\right], \quad \Gamma_{44}=\bar{\gamma}(1-\bar{\gamma}) \tilde{I}^{T} P \tilde{I}-U, \quad \Gamma_{55}=-\gamma^{2} I, \quad \widehat{A}_{1}=\left(\begin{array}{cc}
A_{1} & 0 \\
0 & 0
\end{array}\right) \text {, } \\
& \widehat{A}_{2}=\left(\begin{array}{cc}
A_{2} & 0 \\
0 & 0
\end{array}\right), \quad G_{1}=\operatorname{diag}\left\{\widehat{g}_{1}^{-} \widehat{g}_{1}^{+}, \ldots, \widehat{g}_{n}^{-} \hat{g}_{n}^{+}\right\}, \\
& G_{2}=\operatorname{diag}\left\{\frac{\widehat{g}_{1}^{-}+\widehat{g}_{1}^{+}}{2}, \ldots, \frac{\widehat{g}_{n}^{-}+\widehat{g}_{n}^{+}}{2}\right\} \text {. }
\end{aligned}
$$


Proof. For simplicity, let

$$
\begin{aligned}
h_{1}(k)= & \widetilde{A} \widetilde{x}(k)+\sum_{i=1}^{q} \widetilde{A}_{1 i} \tilde{x}\left(k-\tau_{i}(k)\right) \\
& +\widetilde{A}_{2} \sum_{d=1}^{\infty} \mu_{d} \widetilde{x}(k-d)+\bar{\gamma} \widetilde{I} g(k, x(k))+\widetilde{D} v(k) \\
h_{2}(k)= & \sum_{i=1}^{q} \widetilde{A}_{1 i}(k) \tilde{x}\left(k-\tau_{i}(k)\right) \\
& +\widetilde{A}_{2}(k) \sum_{d=1}^{\infty} \mu_{d} \tilde{x}(k-d)+\widetilde{\gamma}(k) \widetilde{I} g(k, x(k)) .
\end{aligned}
$$

Then, we have the following equation:

$$
\tilde{x}(k+1)=h_{1}(k)+h_{2}(k) \text {. }
$$

For this system, we construct the following Lyapunov functional:

$$
V(k)=V_{1}(k)+V_{2}(k)+V_{3}(k)+V_{4}(k),
$$

with

$$
\begin{gathered}
V_{1}(k)=\tilde{x}^{T}(k) P \tilde{x}(k), \\
V_{2}(k)=\sum_{i=1}^{q} \sum_{j=k-\tau_{i}(k)}^{k-1} \tilde{x}^{T}(j) Q_{i} \tilde{x}(j) \\
V_{3}(k)=\sum_{i=1}^{q} \sum_{j=-\tau_{M}+1}^{-\tau_{m}} \sum_{l=k+j}^{k-1} \tilde{x}^{T}(l) Q_{i} \tilde{x}(l)
\end{gathered}
$$

$V_{4}(k)$

$$
=\sum_{d=1}^{\infty} \sum_{i=k-d}^{k-1} \tilde{x}^{T}(i)\left\{\mu_{d} Q+\mu_{d}^{2} \bar{\beta}(1-\bar{\beta}) \widehat{A}_{2}^{T} P \widehat{A}_{2}\right\} \tilde{x}(i) .
$$

Taking the difference of the functional along the solution of the system, we obtain

$$
\begin{gathered}
\mathbb{E}\{\Delta V(k)\}=\sum_{i=1}^{4} \mathbb{E}\left\{\Delta V_{i}(k)\right\} \\
\mathbb{E}\left\{\Delta V_{1}(k)\right\}=\mathbb{E}\left\{\mathbb{E}\left\{V_{1}(k+1) \mid \tilde{x}_{k}\right\}-V_{1}(k)\right\} \\
=\mathbb{E}\left\{\tilde{x}^{T}(k+1) P \tilde{x}(k+1)-\tilde{x}^{T}(k) P \tilde{x}(k)\right\}
\end{gathered}
$$

$$
\begin{aligned}
&=h_{1}^{T}(k) P h_{1}(k)+\mathbb{E}\left\{2 h_{1}^{T}(k) P h_{2}(k)\right\} \\
&+\mathbb{E}\left\{\sum_{i=1}^{q} \sum_{j=1}^{q} \tilde{x}^{T}\left(k-\tau_{i}(k)\right)\right. \\
& \times \widetilde{A}_{1 i}^{T}(k) P \widetilde{A}_{1 j}(k) \tilde{x}\left(k-\tau_{j}(k)\right) \\
&+\sum_{d_{1}=1}^{\infty}\left[\sum_{d_{2}=1}^{\infty} \mu_{d_{1}} \mu_{d_{2}} \tilde{x}^{T}\left(k-d_{1}\right)\right. \\
&\left.\times \widetilde{A}_{2}^{T}(k) P \widetilde{A}_{2}(k) \tilde{x}\left(k-d_{2}\right)\right] \\
&\left.+\widetilde{\gamma}(k)^{2} g^{T}(k, x(k)) \tilde{I}^{T} P \widetilde{I} g(k, x(k))\right\}
\end{aligned}
$$$$
-\tilde{x}^{T}(k) P \tilde{x}(k) .
$$

It is obvious that

$$
\begin{aligned}
& \mathbb{E}\left\{2 h_{1}^{T}(k) P h_{2}(k)\right\}=0, \\
& \mathbb{E}\left\{\widetilde{A}_{1 i}^{T}(k) P \widetilde{A}_{1 j}(k)\right\}= \begin{cases}0 & i \neq j \\
\bar{\alpha}_{i}\left(1-\bar{\alpha}_{i}\right) \widehat{A}_{1}^{T} P \widehat{A}_{1} & i=j,\end{cases} \\
& \mathbb{E}\left\{\sum_{i=1}^{q} \sum_{j=1}^{q} \widetilde{x}^{T}\left(k-\tau_{i}(k)\right) \widetilde{A}_{1 i}(k) P \widetilde{A}_{1 j}(k) \tilde{x}\left(k-\tau_{j}(k)\right)\right. \\
& +\sum_{d_{1}=1}^{\infty} \sum_{d_{2}=1}^{\infty} \mu_{d_{1}} \mu_{d_{2}} \tilde{x}^{T}\left(k-d_{1}\right) \widetilde{A}_{2}^{T}(k) P \widetilde{A}_{2}(k) \tilde{x}\left(k-d_{2}\right) \\
& \left.+\widetilde{\gamma}(k)^{2} g^{T}(k, x(k)) \widetilde{I}^{T} P \widetilde{I} g(k, x(k))\right\} \\
& =\sum_{i=1}^{q} \tilde{x}^{T}\left(k-\tau_{i}(k)\right) \bar{\alpha}_{i}\left(1-\bar{\alpha}_{i}\right) \widehat{A}_{1}^{T} P \widehat{A}_{1} \tilde{x}\left(k-\tau_{i}(k)\right) \\
& +\sum_{d=1}^{\infty} \tilde{x}^{T}(k-d) \bar{\beta}(1-\tilde{\beta}) \mu_{d}^{2} \widehat{A}_{2}^{T} P \widehat{A}_{2} \tilde{x}(k-d) \\
& +g^{T}(k, x(k)) \bar{\gamma}(1-\bar{\gamma}) \tilde{I}^{T} P \widetilde{I} g(k, x(k)) \text {. }
\end{aligned}
$$

Then, we have the following equations:

$$
\begin{aligned}
\mathbb{E}\left\{\Delta V_{1}(k)\right\}= & h_{1}^{T}(k) P h_{1}(k)-\tilde{x}^{T}(k) P \tilde{x}(k) \\
& +\sum_{i=1}^{q} \tilde{x}^{T}\left(k-\tau_{i}(k)\right) \bar{\alpha}_{i}\left(1-\bar{\alpha}_{i}\right) \\
& \quad \times \widehat{A}_{1}^{T} P \widehat{A}_{1} \tilde{x}\left(k-\tau_{i}(k)\right) \\
& +\sum_{d=1}^{\infty} \widetilde{x}^{T}(k-d) \bar{\beta}(1-\widetilde{\beta}) \mu_{d}^{2} \widehat{A}_{2}^{T} P \widehat{A}_{2} \widetilde{x}(k-d) \\
& +g^{T}(k, x(k)) \bar{\gamma}(1-\bar{\gamma}) \widetilde{I}^{T} P \widetilde{I} g(k, x(k))
\end{aligned}
$$




$$
\begin{aligned}
& \mathbb{E}\left\{\Delta V_{2}(k)\right\}=\mathbb{E}\left\{\mathbb{E}\left\{V_{2}(k+1)\right\}-V_{2}(k)\right\} \\
& =\mathbb{E}\left\{\sum_{i=1}^{q} \sum_{j=k+1-\tau_{i}(k+1)}^{k} \tilde{x}^{T}(j) Q_{i} \tilde{x}(j)\right. \\
& \left.-\sum_{i=1}^{q} \sum_{j=k-\tau_{i}(k)}^{k-1} \tilde{x}^{T}(j) Q_{i} \tilde{x}(j)\right\} \\
& =\sum_{i=1}^{q} \mathbb{E}\left\{\tilde{x}^{T}(k) Q_{i} \tilde{x}(k)\right. \\
& \left.\times\left[\mu_{d} Q+\mu_{d}^{2} \bar{\beta}(1-\bar{\beta}) \widehat{A}_{2}^{T} P \widehat{A}_{2} \tilde{x}(i)\right]\right\} \\
& =\tilde{x}^{T}(k)\left(\bar{\mu} Q+\widehat{\mu} \bar{\beta}(1-\bar{\beta}) \widehat{A}_{2}^{T} P \widehat{A}_{2}\right) \widetilde{x}(k) \\
& -\sum_{d=1}^{\infty} \tilde{x}^{T}(k-d) \mu_{d}^{2} \bar{\beta}(1-\bar{\beta}) \widehat{A}_{2}^{T} P \widehat{A}_{2} \tilde{x} \\
& \times(k-d)-\sum_{d=1}^{\infty} \tilde{x}^{T}(k-d) \mu_{d} Q \tilde{x}(k-d) .
\end{aligned}
$$

$$
\begin{array}{lc}
-\tilde{x}^{T}\left(k-\tau_{i}(k)\right) Q_{i} g\left(k-\tau_{i}(k)\right) & \text { By using Lemma 8, we have } \\
+\sum_{j=k-\tau_{i}(k+1)+1}^{k-1} \tilde{x}^{T}(j) Q_{i} \tilde{x}(j) & -\sum_{d=1}^{\infty} \tilde{x}^{T}(k-d) \mu_{d} Q \tilde{x}(k-d) \\
\left.-\sum_{j=k-\tau_{i}(k)+1}^{k-1} \tilde{x}^{T}(j) Q_{i} \tilde{x}(j)\right\} & \leq-\frac{1}{\mu}\left(\sum_{d=1}^{\infty} \tilde{x}(k-d)\right)^{T} Q\left(\sum_{d=1}^{\infty} \tilde{x}(k-d)\right) .
\end{array}
$$$$
\leq \sum_{i=1}^{q} \mathbb{E}\left\{\tilde{x}^{T}(k) Q_{i} \tilde{x}(k)\right.
$$$$
-\tilde{x}^{T}\left(k-\tau_{i}(k)\right) Q_{i} \tilde{x}\left(k-\tau_{i}(k)\right)
$$$$
\left.+\sum_{j=k-\tau_{M}+1}^{k-\tau_{m}} \tilde{x}^{T}(j) Q_{i} \tilde{x}(j)\right\},
$$$$
\mathbb{E}\left\{\Delta V_{3}(k)\right\}=\mathbb{E}\left\{\mathbb{E}\left\{V_{3}(k+1)\right\}-V_{3}(k)\right\}
$$$$
=\sum_{i=1}^{q} \sum_{j=-\tau_{M}+1}^{-\tau_{m}} \sum_{l=k+j+1}^{k} \tilde{x}^{T}(l) Q_{i} \tilde{x}(l)
$$$$
-\sum_{i=1}^{q} \sum_{j=-\tau_{M}+1}^{-\tau_{m}} \sum_{l=k+j}^{k-1} \tilde{x}^{T}(l) Q_{i} \tilde{x}(l)
$$$$
=\sum_{i=1}^{q}\left(\left(\tau_{M}-\tau_{m}\right) \tilde{x}^{T}(k) Q_{i} \tilde{x}(k)\right.
$$$$
\left.-\sum_{j=k-\tau_{M}-1}^{k-\tau_{m}} \tilde{x}^{T}(j) Q_{i} \tilde{x}(j)\right),
$$$$
\mathbb{E}\left\{\Delta V_{4}(k)\right\}=\mathbb{E}\left\{\mathbb{E}\left\{V_{4}(k+1)\right\}-V_{4}(k)\right\}
$$

$$
\begin{aligned}
=\sum_{d=1}^{\infty} \mathbb{E} & \left\{\sum_{i=k-d+1}^{k} \tilde{x}^{T}(i)\right. \\
& \times\left[\mu_{d} Q+\mu_{d}^{2} \bar{\beta}(1-\bar{\beta}) \widehat{A}_{2}^{T} P \widehat{A}_{2}\right] \tilde{x}(i) \\
& -\sum_{i=k-d}^{k-1} \tilde{x}^{T}(i)
\end{aligned}
$$

From Assumption 1, $\widehat{g}_{i}^{-} \leq\left(g_{i}(x)-g_{i}(y)\right) /(x-y) \leq \hat{g}_{i}^{+}$, we have $\left(g(k, x(k))-\widehat{g}_{i}^{-} x(k)\right)\left(g(k, x(k))-\widehat{g}_{i}^{+} x(k)\right) \leq 0$.

It can be deduced that there exists $\mathbf{U}=$ $\operatorname{diag}\left[u_{1}, u_{2}, \ldots, u_{n}\right]>0$ such that

$$
\begin{gathered}
\sum_{i=1}^{n} u_{i}\left[\begin{array}{c}
x(k) \\
g(k)
\end{array}\right]^{T}\left[\begin{array}{cc}
\hat{g}_{i}^{-} \hat{g}_{i}^{+} e_{i} e_{i}^{T} & -\frac{\hat{g}_{i}^{-}+\hat{g}_{i}^{+}}{2} e_{i} e_{i}^{T} \\
-\frac{\hat{g}_{i}^{-}+\hat{g}_{i}^{+}}{2} e_{i} e_{i}^{T} & e_{i} e_{i}^{T}
\end{array}\right] \\
\quad \times\left[\begin{array}{l}
x(k) \\
g(k)
\end{array}\right] \\
=\left[\begin{array}{c}
x(k) \\
g(k)
\end{array}\right]^{T}\left[\begin{array}{cc}
U G_{1} & -U G_{2} \\
-U G_{2} & U
\end{array}\right]\left[\begin{array}{l}
x(k) \\
g(k)
\end{array}\right] \leq 0,
\end{gathered}
$$

where $e_{i}$ denotes the unit column vector having one element on its $r$ th row and zeros elsewhere.

Now, we are ready to prove the exponential stability of the system (8) with $v(k)=0$. Combining (16)-(24), we can easily see that

$$
\mathbb{E}\{\Delta V(k)\} \leq \mathbb{E}\left\{\zeta_{1}^{T}(k)\left(\Gamma_{1}+\Delta^{T} P \Delta\right) \zeta_{1}(k)\right\}
$$

where 


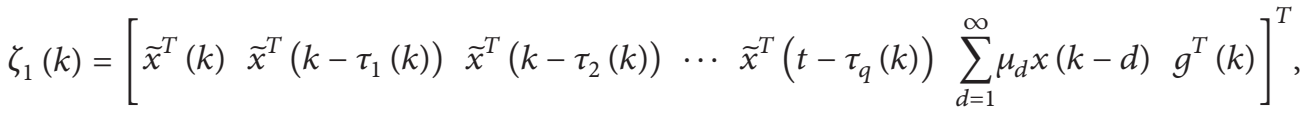

$$
\begin{aligned}
& \Gamma_{1}=\left[\begin{array}{cccccc}
\phi_{11} & 0 & \cdots & 0 & 0 & 0 \\
* & \bar{\alpha}_{1}\left(1-\bar{\alpha}_{1}\right) \widehat{A}_{1}^{T} P \widehat{A}_{1}-Q_{1} & \cdots & 0 & 0 & 0 \\
* & * & \ddots & \vdots & \vdots & \vdots \\
* & * & * & \bar{\alpha}_{q}\left(1-\bar{\alpha}_{q}\right) \widehat{A}_{1}^{T} P \widehat{A}_{1}-Q_{q} & 0 & 0 \\
* & * & * & * & -\frac{Q}{\bar{\mu}} & 0 \\
* & * & * & * & * & \bar{\gamma}(1-\bar{\gamma}) \widetilde{I}^{T} P \widetilde{I}-l I
\end{array}\right] \\
& \Delta=\left[\begin{array}{llllll}
\widetilde{A} & \widetilde{A}_{11} & \cdots & \widetilde{A}_{1 q} & \widetilde{A}_{2} & \bar{\gamma} \widetilde{I}
\end{array}\right] .
\end{aligned}
$$

If $\Gamma_{1}+\Delta^{T} P \Delta<0$, we can obtain that $\Gamma_{2}<0$ by Schur complements (Lemma 7), where

$$
\Gamma_{2}=\left[\begin{array}{ccccccc}
\phi_{11} & 0 & \cdots & 0 & 0 & 0 & \widetilde{A}^{T} P \\
* & \bar{\alpha}_{1}\left(1-\bar{\alpha}_{1}\right) \widehat{A}_{1}^{T} P \widehat{A}_{1}-Q_{1} & \cdots & 0 & 0 & 0 & \widetilde{A}_{11}^{T} P \\
* & * & \ddots & \vdots & \vdots & \vdots & \vdots \\
* & * & * & \bar{\alpha}_{q}\left(1-\bar{\alpha}_{q}\right) \widehat{A}_{1}^{T} P \widehat{A}_{1}-Q_{q} & 0 & 0 & \widetilde{A}_{1 q}^{T} P \\
* & * & * & * & -\frac{Q}{\bar{\mu}} & 0 & \widetilde{A}_{2}^{T} P \\
* & * & * & * & * & & * \\
* & * & * & * & * & * & -P
\end{array}\right] .
$$

Then, the system (8) with $v(k)=0$ is mean-square robustly asymptotically stable. Furthermore, along the same line of the proof for Theorem 1 in [15], the exponential stability of system (8) can be confirmed on the mean-square sense.

Next, we will establish the performance of the filtering error system (8) under the zero initial condition. To this end, we introduce

$$
\begin{aligned}
J(k)= & E \sum_{k=0}^{\infty}\left\{\tilde{r}^{T}(k) \widetilde{r}(k)\right\} \\
& -\gamma^{2} \sum_{k=0}^{\infty} E\left\{v^{T}(k) v(k)\right\} .
\end{aligned}
$$

$$
\begin{aligned}
J(n)= & E \sum_{k=0}^{n}\left\{\widetilde{r}^{T}(k) \widetilde{r}(k)\right\} \\
& -\gamma^{2} \sum_{k=0}^{n} E\left\{v^{T}(k) v(k)\right\} \\
& +E \sum_{k=0}^{n}\{V(k+1)-V(k)\}-V(n+1)+V(0) \\
\leq & E \sum_{k=0}^{n}\left\{\widetilde{r}^{T}(k) \widetilde{r}(k)\right\}-\gamma^{2} \sum_{k=0}^{n} E\left\{v^{T}(k) v(k)\right\} \\
& +E \sum_{k=0}^{n}\{\Delta V(k)\} .
\end{aligned}
$$

Along the same line as the proof of the stability of system (7), $J(n) \leq E \sum_{k=0}^{n}\left\{\zeta_{2}^{T}(k) \Gamma \zeta_{2}(k)\right\}$, where

And, it is obvious to see that

$$
\zeta_{2}(k)=\left[\begin{array}{llllllll}
\tilde{x}^{T}(k) & \tilde{x}^{T}\left(k-\tau_{1}(k)\right) & \tilde{x}^{T}\left(k-\tau_{2}(k)\right) & \cdots & \tilde{x}^{T}\left(t-\tau_{q}(k)\right) & \sum_{d=1}^{\infty} \mu_{d} x^{T}(k-d) & g^{T}(k) & v^{T}(k)
\end{array}\right]^{T},
$$

we can show that $J(n) \leq 0$. 
Letting $n \rightarrow \infty$, we obtain

$$
\sum_{k=0}^{+\infty} E\left\{\tilde{r}^{T}(k) \tilde{r}(k)\right\} \leq \gamma^{2} \sum_{k=0}^{\infty} E\left\{v^{T}(k) v(k)\right\} .
$$

This completes the proof.

Next, we are in a position to deal with the design of the filter output feedback controller for the system (8) and obtain the main result of this paper in the following theorem.
Theorem 10. For given constants $0 \leq \tau_{m}<\tau_{M}, \varepsilon>0$, and a scalar $\lambda>0$, the fault detection filter (7) exists such that error system (8) is robustly asymptotically exponentially stable in mean square, if there exist constant $l>0$, positive matrices $P, R$, and $R_{i}(i=1,2, \ldots, q)$, and any matrices $\widehat{A}_{f}, \widehat{B}_{f}, \widehat{C}_{f}$, and $\widehat{D}_{f}$ with appropriate dimensions, respectively,

$$
\begin{aligned}
& \Sigma=\left[\begin{array}{ccccccccc}
\Sigma_{11} & 0 & \cdots & 0 & 0 & \Sigma_{14} & 0 & \Sigma_{16} & \Sigma_{17} \\
* & \bar{\alpha}_{1}\left(1-\bar{\alpha}_{1}\right) \bar{A}_{1}-R_{1} & \cdots & 0 & 0 & 0 & 0 & 0 & \Sigma_{2,17} \\
* & * & \ddots & \vdots & \vdots & \vdots & \vdots & \vdots & \vdots \\
* & * & * & \bar{\alpha}_{q}\left(1-\bar{\alpha}_{q}\right) \bar{A}_{1}-R_{q} & 0 & 0 & 0 & 0 & \Sigma_{2, q 7} \\
* & * & * & * & -\frac{R}{\bar{\mu}} & 0 & 0 & 0 & \Sigma_{37} \\
* & * & * & * & * & \Sigma_{44} & 0 & 0 & \Sigma_{47} \\
* & * & * & * & * & * & \Sigma_{55} & \Sigma_{56} & \Sigma_{57} \\
* & * & * & * & * & * & * & -I & 0 \\
* & * & * & * & * & * & * & * & \Sigma_{7}
\end{array}\right]<0, \\
& \Sigma_{11}=-\left(\begin{array}{cc}
X & Y \\
Y & Y
\end{array}\right)+\sum_{i=1}^{q}\left(\tau_{M}-\tau_{m}+1\right) R_{i}+\bar{\mu} R+\widehat{\mu} \bar{\beta}(1-\bar{\beta}) \bar{A}_{2}^{T}-\widetilde{U}, \\
& \widetilde{U}=\left(\begin{array}{cc}
U G_{1} & U G_{1} \\
U G_{1} & U G_{1}
\end{array}\right), \quad \bar{A}_{1}=\left(\begin{array}{cc}
A_{1}^{T} X A_{1} & A_{1}^{T} X A_{1} \\
A_{1}^{T} X A_{1} & A_{1}^{T} X A_{1}
\end{array}\right), \quad \bar{A}_{2}=\left(\begin{array}{cc}
A_{2}^{T} X A_{2} & A_{2}^{T} X A_{2} \\
A_{2}^{T} X A_{2} & A_{2}^{T} X A_{2}
\end{array}\right), \\
& \Sigma_{14}=\left[\begin{array}{c}
U G_{2} \\
U G_{2}
\end{array}\right], \quad \Sigma_{16}=\left[\begin{array}{ll}
C^{T} \widehat{D}_{f}^{T} & C^{T} \widehat{D}_{f}^{T}+\widehat{C}_{f}^{T}
\end{array}\right], \quad \Sigma_{17}=\left[\begin{array}{cc}
A^{T} X+C^{T} \widehat{B}_{f}^{T} & A^{T} Y \\
A^{T} X+C^{T} \widehat{B}_{f}^{T}+\widehat{A}_{f}^{T} & A^{T} Y
\end{array}\right], \\
& \Sigma_{2, i 7}=\left[\begin{array}{cc}
\bar{\alpha}_{i} A_{1}^{T} X & \bar{\alpha}_{i} A_{1}^{T} Y \\
\bar{\alpha}_{i} A_{1}^{T} X & \bar{\alpha}_{i} A_{1}^{T} Y
\end{array}\right] \quad(i=1,2, \ldots, q), \\
& \Sigma_{37}=\left[\begin{array}{ll}
\bar{\beta} A_{2} X & \bar{\beta} A_{2} Y \\
\bar{\beta} A_{2} X & \bar{\beta} A_{2} Y
\end{array}\right], \quad \Sigma_{44}=\bar{\gamma}(1-\bar{\gamma}) X-U, \quad \Sigma_{55}=-\gamma^{2} I, \quad \Sigma_{47}=\left[\begin{array}{ll}
\gamma X & \gamma Y
\end{array}\right], \\
& \Sigma_{56}=\left[\begin{array}{c}
D_{2}^{T} \widetilde{D}_{f}^{T} \\
H^{T} \widetilde{D}_{f}^{T}-I
\end{array}\right], \quad \Sigma_{57}=\left[\begin{array}{cc}
D_{1}^{T} X+D_{2}^{T} \widehat{B}_{f}^{T} & D_{1}^{T} Y \\
G^{T} X+H^{T} \widehat{B}_{f}^{T} & G^{T} Y
\end{array}\right], \quad \Sigma_{77}=-\left[\begin{array}{cc}
X & Y \\
Y & Y
\end{array}\right] .
\end{aligned}
$$

If $\left(X, Y, Q, Q_{i}\right)$ is a feasible solution of (15), there exist nonsingular matrices $U$ and $V$ satisfying $U V=I-X Y^{-1}$. The fault detection filter parameters in the form of (7) are given as follows:

$$
A_{f}=U^{-1} \widehat{A}_{f} Y^{-1} V^{-1}, \quad B_{f}=U^{-1} \widehat{B}_{f},
$$

$$
C_{f}=\widehat{C}_{f} Y^{-1} V^{-1}, \quad D_{f}=\widehat{D}_{f}
$$

Proof. The condition in (31) pledges that $\left(\begin{array}{ll}X & Y \\ * & Y\end{array}\right)>0$.

This inequality implies that $I-X Y^{-1}$ and $X-Y$ are nonsingular by Schur complement. If the condition in (32) 
is feasible, there exist nonsingular matrices $U, V$ satisfying $U V=I-X Y^{-1}$.

Then, we introduce the following matrices:

$$
\Omega_{1}=\left(\begin{array}{cc}
X & Y \\
U^{T} & 0
\end{array}\right), \quad \Omega_{2}=\left(\begin{array}{cc}
I & I \\
0 & V Y
\end{array}\right)
$$

Obviously $\Omega_{1}, \Omega_{2}$ are nonsingular.

Let

$$
P \triangleq \Omega_{1} \Omega_{2}^{-1}=\left(\begin{array}{cc}
X & U \\
U^{T} & -U^{T} Y^{-1} V^{-1}
\end{array}\right)
$$

then $\Omega_{2}^{T} P \Omega_{2}=\left(\begin{array}{cc}X & Y \\ Y^{T} & Y\end{array}\right)$.
Pre- and postmultiplying inequality (32) by $\operatorname{diag}\left\{\Omega_{2}^{-1}\right.$, $k \underbrace{\Omega_{2}^{-1}, \ldots, \Omega_{2}^{-1}}_{q}, \Omega_{2}^{-1}, I, I, I, \Omega_{2}^{-1}\}$, and letting $\Omega_{2}^{T} Q_{i} \Omega_{2}=R_{i}$, $\Omega_{2}^{T} Q \Omega_{2}=R$, we can obtain (8). This com- pletes the proof.

When there are parameters uncertainties, we have the following corollary.

Corollary 11. For given constants $0 \leq \tau_{m}<\tau_{M}, \varepsilon>0$ and a scalar $\lambda>0$, the fault detection filter (7) exists such that error system (8) is robustly asymptotically exponentially stable in mean square, if there exist constant $l>0, \epsilon>0$, positive matrices $P, R$, and $R_{i}(i=1,2, \ldots, q)$, and any matrices $\widehat{A}_{f}$, $\widehat{B}_{f}, \widehat{C}_{f}$, and $\widehat{D}_{f}$ with appropriate dimensions, respectively,

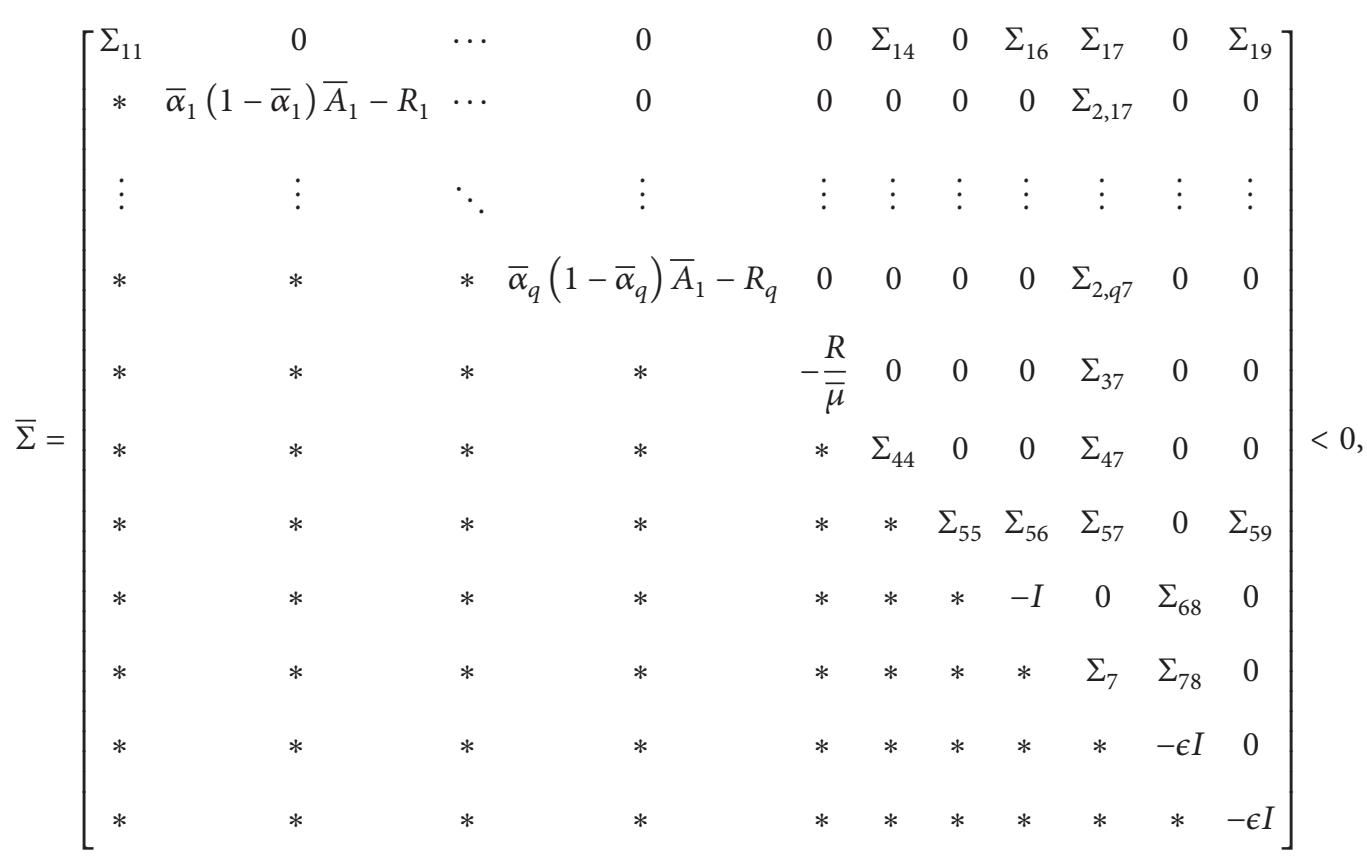

where, $\Sigma_{11}, \Sigma_{16}, \Sigma_{17}, \Sigma_{2,17}, \ldots, \Sigma_{2, q 7}, \bar{A}_{1}, \Sigma_{37}, \Sigma_{44}, \Sigma_{47}, \Sigma_{55}, \Sigma_{56}$, $\Sigma_{57}$, and $\Sigma_{7}$ have been defined in Theorem 9:

$$
\begin{gathered}
\Sigma_{19}=\left[\begin{array}{c}
C^{T} \Lambda^{T} \\
C^{T} \Lambda^{T}
\end{array}\right], \quad \Sigma_{59}=\left[\begin{array}{c}
D_{2}^{T} \Lambda^{T} \\
H^{T} \Lambda^{T}
\end{array}\right], \\
\Sigma_{68}=\left[\epsilon \widehat{D}_{f}\right], \quad \Sigma_{78}=-\left[\begin{array}{c}
\epsilon \widehat{B}_{f} \\
0
\end{array}\right] .
\end{gathered}
$$

If $\left(X, Y, Q, Q_{i}\right)$ is a feasible solution of (15), there exist nonsingular matrices $U$ and $V$ satisfying $U V=I-X Y^{-1}$. The fault detection filter parameters in the form of (7) are given as follows:

$$
\begin{array}{clrl}
A_{f} & =U^{-1} \widehat{A}_{f} Y^{-1} V^{-1}, & B_{f} & =U^{-1} \widehat{B}_{f}, \\
C_{f}=\widehat{C}_{f} Y^{-1} V^{-1}, & D_{f} & =\widehat{D}_{f} .
\end{array}
$$

Proof. When considering the system with uncertain parameters, we need to replace $\widetilde{A}, \widetilde{D}, \widetilde{C}, \widetilde{D} f$ with $\widetilde{A}+\Delta A, \widetilde{D}+\Delta D, \widetilde{C}+$ $\Delta C, \widetilde{D}_{f}+\Delta D_{f}$ in (32). Then, (32) changes into $\Sigma+M F(k) N+$ $N^{T} F^{T}(k) M^{T}<0$, where $M=\left(0,0, \ldots, 0, \widehat{D}_{f}^{T}, \widehat{B}_{f}^{T}, 0\right)^{T} ; N=$ $\left(\Lambda C, \Lambda C, 0, \ldots, 0, \Lambda D_{2}, \Lambda H, 0,0,0\right)$.

Using Schur complement and lemma, we can derive (37). This completes the proof.

\section{Illustrative Example}

In this section, a numerical example will be presented to illustrate the effectiveness of our results.

Example 1. Consider the system (2) with the parameters listed as follows [13]:

$$
A=\left[\begin{array}{cc}
0.9719 & -0.0013 \\
-0.0340 & 0.8628
\end{array}\right], \quad A_{1}=\left[\begin{array}{cc}
0.14 & 0.2 \\
0 & 0.2
\end{array}\right],
$$




$$
\begin{gathered}
A_{2}=\left[\begin{array}{ll}
0 & 0 \\
0 & 0
\end{array}\right], \quad D_{1}=\left[\begin{array}{cc}
0.1 & 0 \\
0 & 0.3
\end{array}\right], \\
D_{2}=\left[\begin{array}{ll}
0 & 0.1
\end{array}\right], \quad C=\left[\begin{array}{cc}
1 & 0.1
\end{array}\right], \\
G=\left[\begin{array}{ll}
-0.0839 & 0.0761
\end{array}\right]^{T}, \quad B=0, \\
H=0, \quad \bar{\alpha}_{1}=0.9, \quad \bar{\alpha}_{2}=0.7, \\
\bar{\gamma}=0.8, \quad g_{1}(k, x(k))=0.5 x_{1}(k) \sin \left(x_{2}(k)\right), \\
g_{2}(k, x(k))=0.5 x_{2}(k) \sin \left(x_{1}(k)\right), \quad \varepsilon(k)=1, \\
G_{1}=\left[\begin{array}{cc}
0.25 & 0 \\
0 & 0.26
\end{array}\right], \quad G_{2}=\left[\begin{array}{cc}
0.45 & 0 \\
0 & 0.45
\end{array}\right] .
\end{gathered}
$$

Let the time-varying communication delays satisfy $1 \leq$ $\tau_{i}(k) \leq 3(i=1,2)$. For the measurement quantization, the parameters of the logarithmic quantizer are set as $\widehat{\mu}_{0}=2$ and $\chi=0.8$. Then, the fault detection filter parameters can be obtained from Corollary 11 as follows.

For the parameters listed previously, by Corollary 11 in our paper, we can obtain the following feasible filtering parameters:

$$
\begin{gathered}
A_{f}=\left[\begin{array}{ll}
-0.2524 & -0.0325 \\
-0.0325 & -0.1975
\end{array}\right], \quad B_{f}=\left[\begin{array}{l}
-3.4252 \\
-0.4137
\end{array}\right], \\
C_{f}=\left[\begin{array}{ll}
0.0065 & -0.0045
\end{array}\right], \quad D_{f}=[0.0122],
\end{gathered}
$$

and the performance index between the robustness and sensitivity is $\gamma=3.8382$.

Example 2. To show the usefulness and effectiveness of the designed fault detection filter, let the external disturbance be $w(k)=0$. Figures 1 and 2 show the residual signal $r(k)$ and evolution of residual function $J(k)$, respectively, when the fault signal $f(k)$ is given as

$$
f(k)= \begin{cases}1, & 40 \leq k \leq 80 \\ 0, & \text { else. }\end{cases}
$$

It indicates that the designed filter can detect the fault effectively. In [13], the fault can be detected in 6 time steps after its occurrence. However, from Figure 2, we can see that the fault can be detected in 4 time steps after its occurrence. Therefore, it can be seen that the residual can not only reflect the fault in time but also detect the fault without confusing it with the disturbance $w(k)$.

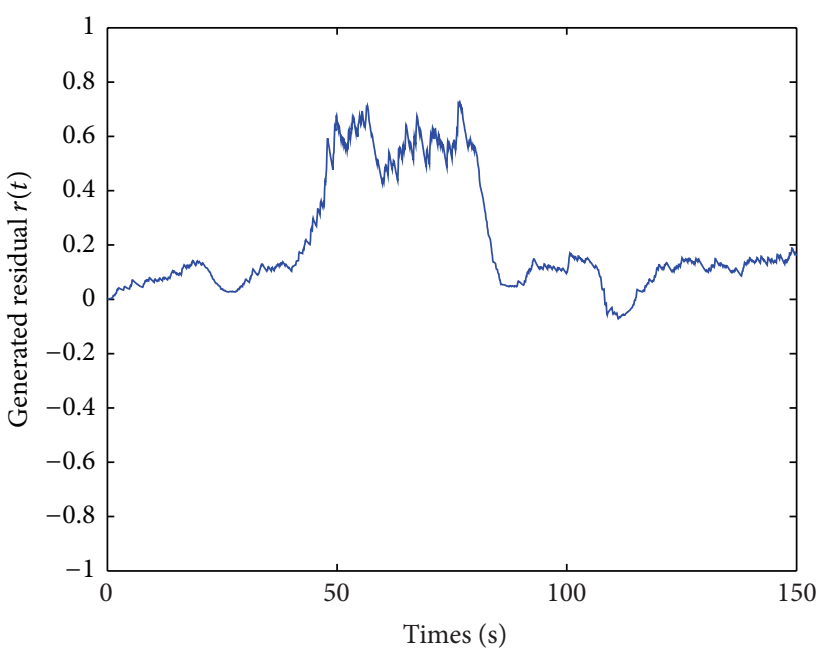

FIgURE 1: Residual signal without $w(k)$.

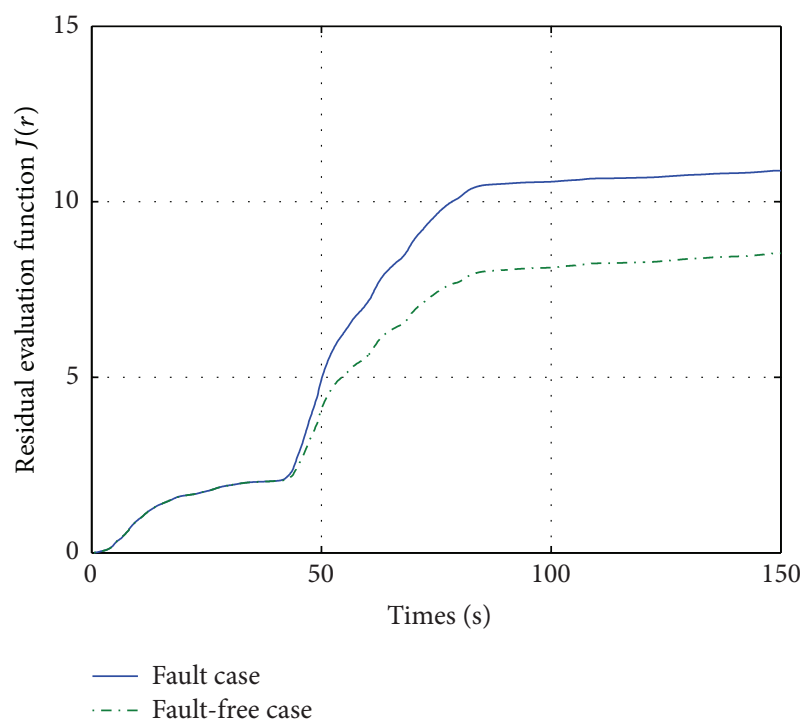

FIgURE 2: Evolution of residual function $J(k)$ without $w(k)$.

\section{Conclusion}

The fault detection filter design for stochastic systems with time-varying delays has been investigated in this research. Based on the Lyapunov functional method, sufficient conditions are obtained to ensure that the error systems are meansquare robustly asymptotically stable, and then the filters are designed in terms of LMIs. Numerical example has been given to illustrate the effectiveness of the proposed main results. The foregoing results have the potential to be useful for the study of stochastic systems.

\section{Acknowledgments}

This research was supported by the National Basic Research Program of China (2010 CB732501), the Fund of Sichuan 
Provincial Key Laboratory of Signal and Information Processing (SZJJ2009-002), and the Fund of Sichuan Provincial Key Laboratory of Signal and Information Processing (SGXZD0101-10-1).

\section{References}

[1] S. Zhou and G. Feng, " $H_{\infty}$ filtering for discrete-time systems with randomly varying sensor delays," Automatica, vol. 44, no. 7, pp. 1918-1922, 2008.

[2] H. Gao and C. Wang, "Delay-dependent robust $H_{\infty}$ and $\mathscr{L}_{2}-$ $\mathscr{L}_{\infty}$ filtering for a class of uncertain nonlinear time-delay systems," IEEE Transactions on Automatic Control, vol. 48, no. 9, pp. 1661-1665, 2003.

[3] E. Fridman, U. Shaked, and L. Xie, "Robust $H_{\infty}$ filtering of linear systems with time-varying delay," IEEE Transactions on Automatic Control, vol. 48, no. 1, pp. 159-165, 2003.

[4] H. Li and M. Fu, "Linear matrix inequality approach to robust $H_{\infty}$ filtering," IEEE Transactions on Signal Processing, vol. 45, no. 9, pp. 2338-2350, 1997.

[5] Y. Liu, Z. Wang, J. Liang, and X. Liu, "Synchronization and state estimation for discrete-time complex networks with distributed delays," IEEE Transactions on Systems, Man, and Cybernetics B, vol. 38, no. 5, pp. 1314-1325, 2008.

[6] K. M. Grigoriadis and J. T. Watson Jr., "Reduced-order $H_{\infty}$ and $\mathscr{L}_{2}-\mathscr{L}_{\infty}$ filtering via linear matrix inequalities," IEEE Transactions on Aerospace and Electronic Systems, vol. 33, no. 4, pp. 1326-1338, 1997.

[7] P. M. Frank, "Analytical and qualitative model-based fault diagnosis-a survey and some new results," European Journal of Control, vol. 2, no. 1, pp. 6-28, 1996.

[8] P. M. Frank, "Fault diagnosis in dynamic systems using analytical and knowledge-based redundancy. A survey and some new results," Automatica, vol. 26, no. 3, pp. 459-474, 1990.

[9] I. Daubechies, "Wavelet transform, time-frequency localization and signal analysis," IEEE Transactions on Information Theory, vol. 36, no. 5, pp. 961-1005, 1990.

[10] Z. Wang and D. W. C. Ho, "Filtering on nonlinear time-delay stochastic systems," Automatica, vol. 39, no. 1, pp. 101-109, 2003.

[11] X. Zhang and Q. Han, "Robust $H_{\infty}$ filtering for a class of uncertain linear systems with time-varying delay," Automatica, vol. 44, no. 1, pp. 157-166, 2008.

[12] S. Xu, J. Lam, H. Gao, and Y. Zou, "Robust $H_{\infty}$ filtering for uncertain discrete stochastic systems with time delays," Circuits, Systems, and Signal Processing, vol. 24, no. 6, pp. 753-770, 2005.

[13] H. Dong, Z. Wang, and H. Gao, "On design of quantized fault detection filters with randomly occurring nonlinearities and mixed time-delays," Signal Processing, vol. 92, no. 4, pp. 1117$1125,2012$.

[14] S. Xu and T. Chen, "Robust $H_{\infty}$ filtering for uncertain stochastic time-delay systems," Asian Journal of Control, vol. 5, no. 3, pp. 364-373, 2003.

[15] Z. Wang, G. Wei, and G. Feng, "Reliable $H_{\infty}$ control for discrete-time piecewise linear systems with infinite distributed delays," Automatica, vol. 45, no. 12, pp. 2991-2994, 2009.

[16] Y. Chen and A.-K. Xue, "Improved stability criterion for uncertain stochastic delay systems with nonlinear uncertainties," Electronics Letters, vol. 44, no. 7, pp. 458-459, 2008.

[17] W. Chen, Z. Guan, and X. Lu, "Delay-dependent exponential stability of uncertain stochastic systems with multiple delays: an
LMI approach," Systems and Control Letters, vol. 54, no. 6, pp. 547-555, 2005.

[18] M. Luo, G. Liu, and S. Zhong, "Robust fault detection of Markovian jump systems with different system modes," Applied Mathematical Modelling, vol. 37, no. 7, pp. 5001-5012, 2013.

[19] M. Fu and L. Xie, "The sector bound approach to quantized feedback control," IEEE Transactions on Automatic Control, vol. 50, no. 11, pp. 1698-1711, 2005. 


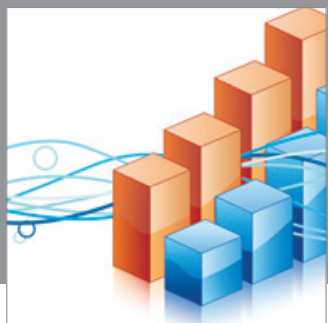

Advances in

Operations Research

mansans

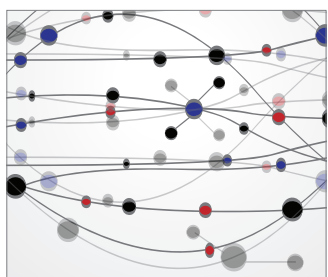

The Scientific World Journal
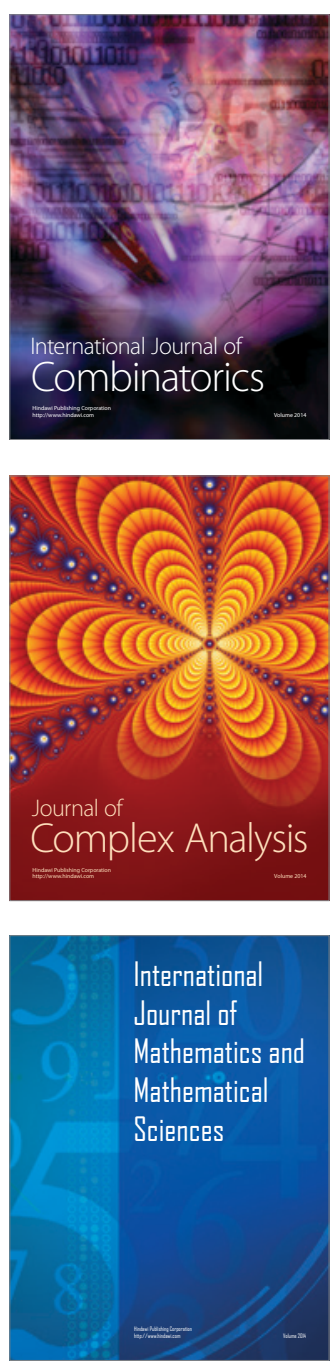
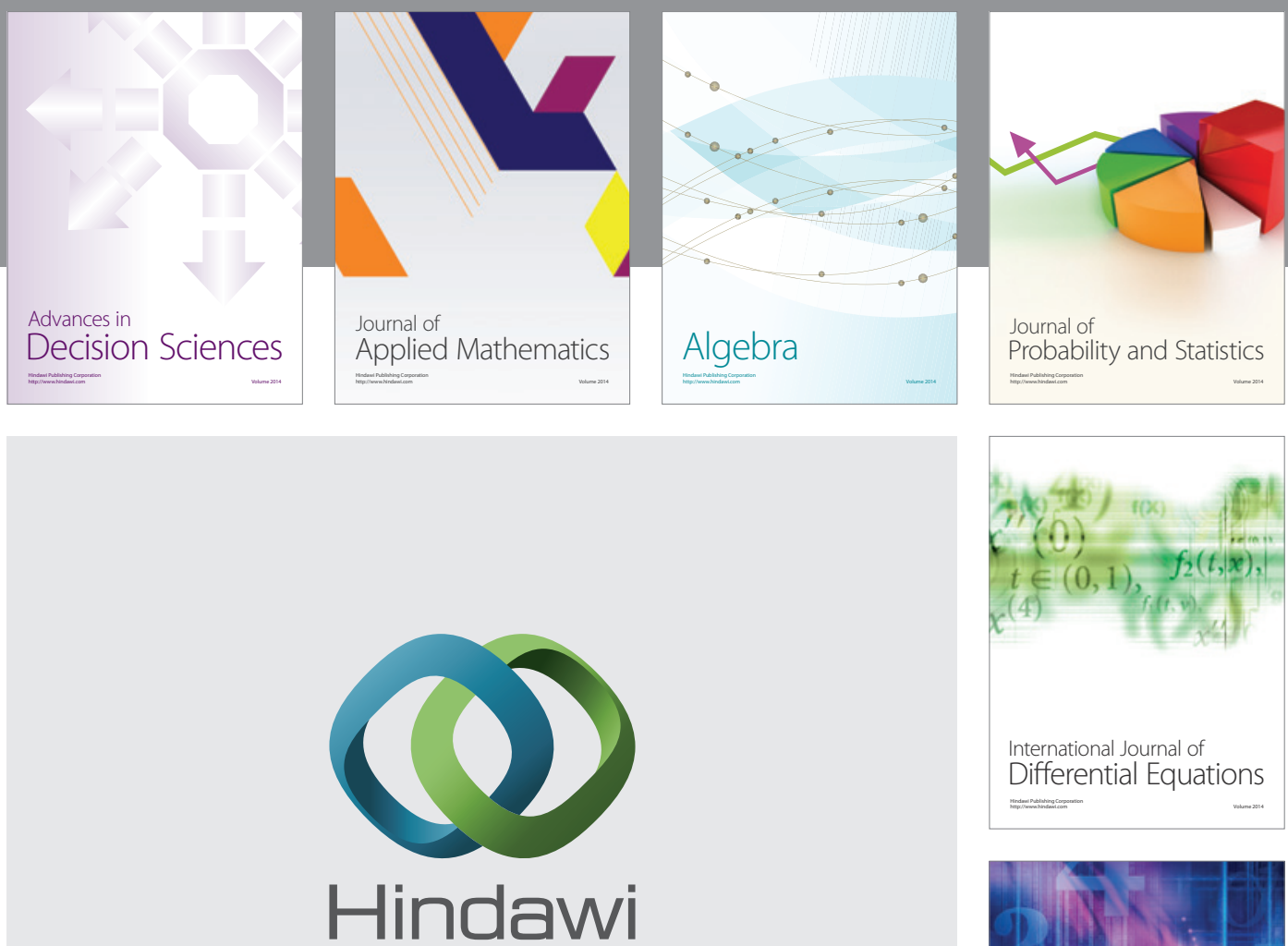

Submit your manuscripts at http://www.hindawi.com
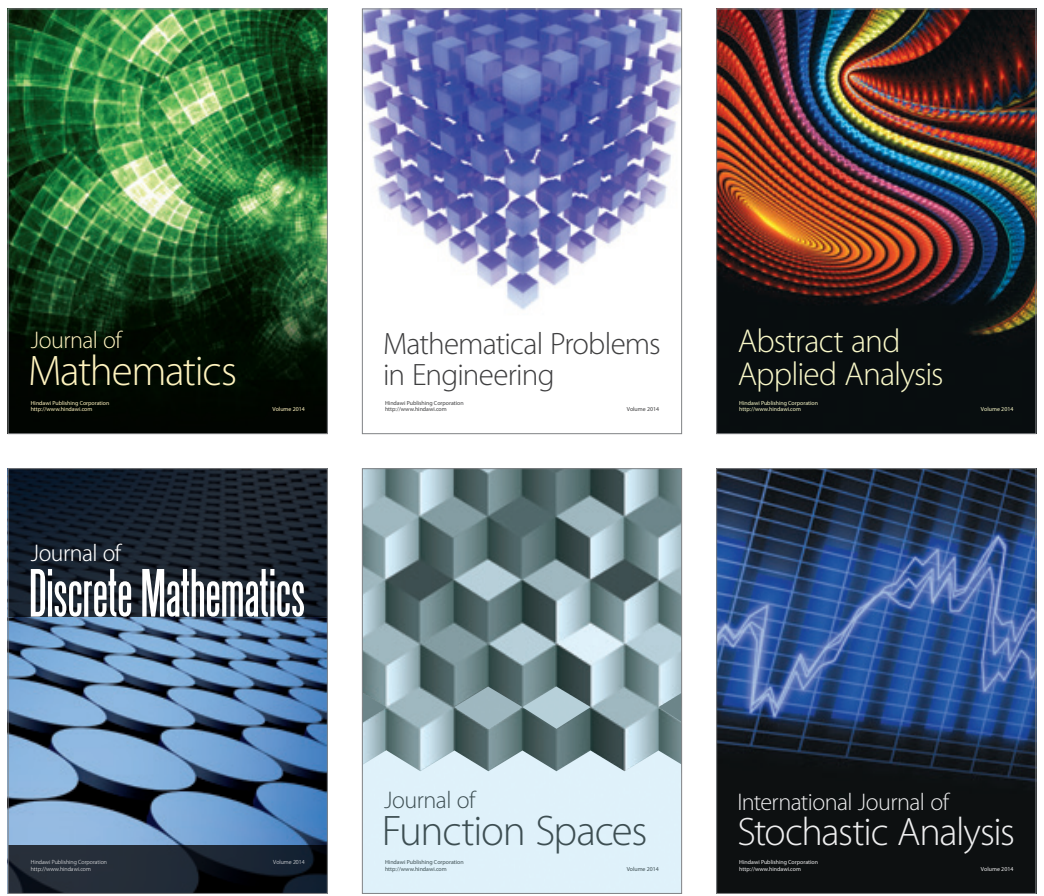

Journal of

Function Spaces

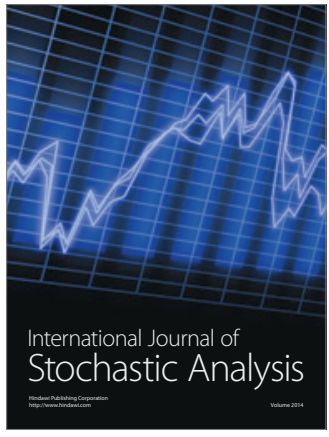

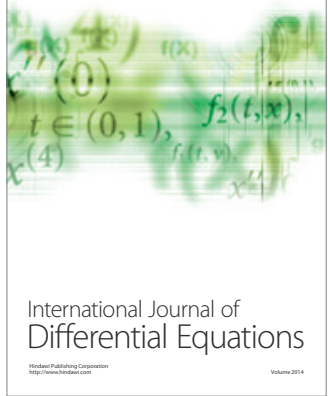
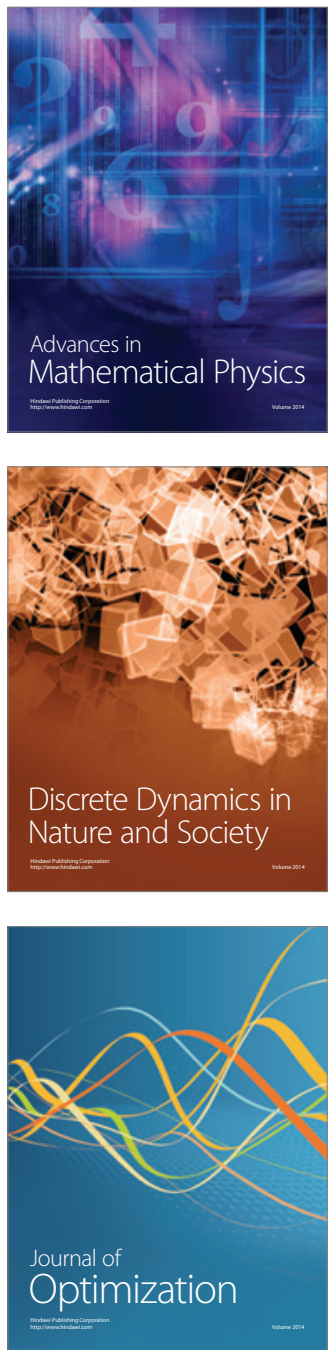